\title{
Homogeneous Studies of Transiting Planets
}

\author{
John Southworth \\ Astrophysics Group, Keele University, Staffordshire, ST5 5BG, UK \\ email: jkt@astro.keele.ac.uk
}

\begin{abstract}
The derived physical properties of the known transiting extrasolar planetary systems come from a variety of sources, and are calculated using a range of different methods so are not always directly comparable. I present a catalogue of the physical properties of 58 transiting extrasolar planet and brown dwarf systems which have been measured using homogeneous methods, resulting in quantities which are internally consistent and well-suited to detailed statistical study. The main results for each object, plus a critical compilation of literature values for all known systems, have been placed in an online catalogue. TEPCat can be found at http://www.astro.keele.ac.uk/ jkt/tepcat/
\end{abstract}

Keywords. stars: planetary systems — stars: fundamental parameters

\section{The Homogeneous Studies project}

At this point roughly 130 transiting extrasolar planets (TEPs) are known, discovered by over 20 different groups and consortia. The characterisation of these objects is complicated by the fact that the number of measured quantities needed to calculate their physical properties is one greater than the number available directly through photometric and spectroscopic observations. This leads to the requirement to include additional constraints, typically from theoretical stellar models, in order to arrive at a determinate solution. The intricacy of this process leads expectedly to an inhomogeneity in the resulting solutions, and hinders statistical studies of these objects. In order to nullify this problem I am undertaking a project to measure the physical properties of the known TEPs by applying homogeneous methods to published data.

The transit light curves are modelled using the JKTEBOP code (Southworth et al., 2004ab), which represents the star and planet as biaxial spheroids. Careful attention is paid to the calculation of robust statistical errors using Monte Carlo simulations (Southworth et al., 2004c, 2005b), the assessment of systematic errors by a residual-permutation algorithm (Jenkins et al., 2002), issues related to the treatment of limb darkening, contaminating 'third' light, and numerical integration over long exposure times. These aspects are discussed in detail in Papers I and III (Southworth 2008, 2010). New transit light curves for five TEPs have also been obtained in the course of a defocussed-photometry project (Southworth et al., 2009abc, 2010, 2011).

Determination of the physical properties of the TEPs is achieved by using each of five different sets of tabulated predictions from theoretical stellar models as constraints. The input quantities are the orbital velocity amplitude, spectroscopic temperature and metallicity of the parent star, plus the results calculated from the light curve solutions. The output parameters comprise the physical properties of the system (Paper II: Southworth 2009). Statistical errors are supplied via a propagation analysis (Southworth et al., 2005a) and systematic errors from consideration of the spread of results obtained using the five different stellar model predictions. Three quantities are free of systematic errors: the mean density of the star (Seager \& Mallén-Ornela 2003), and the surface gravity and equilibrium temperature of the planet (Southworth et al., 2007, Paper III). 


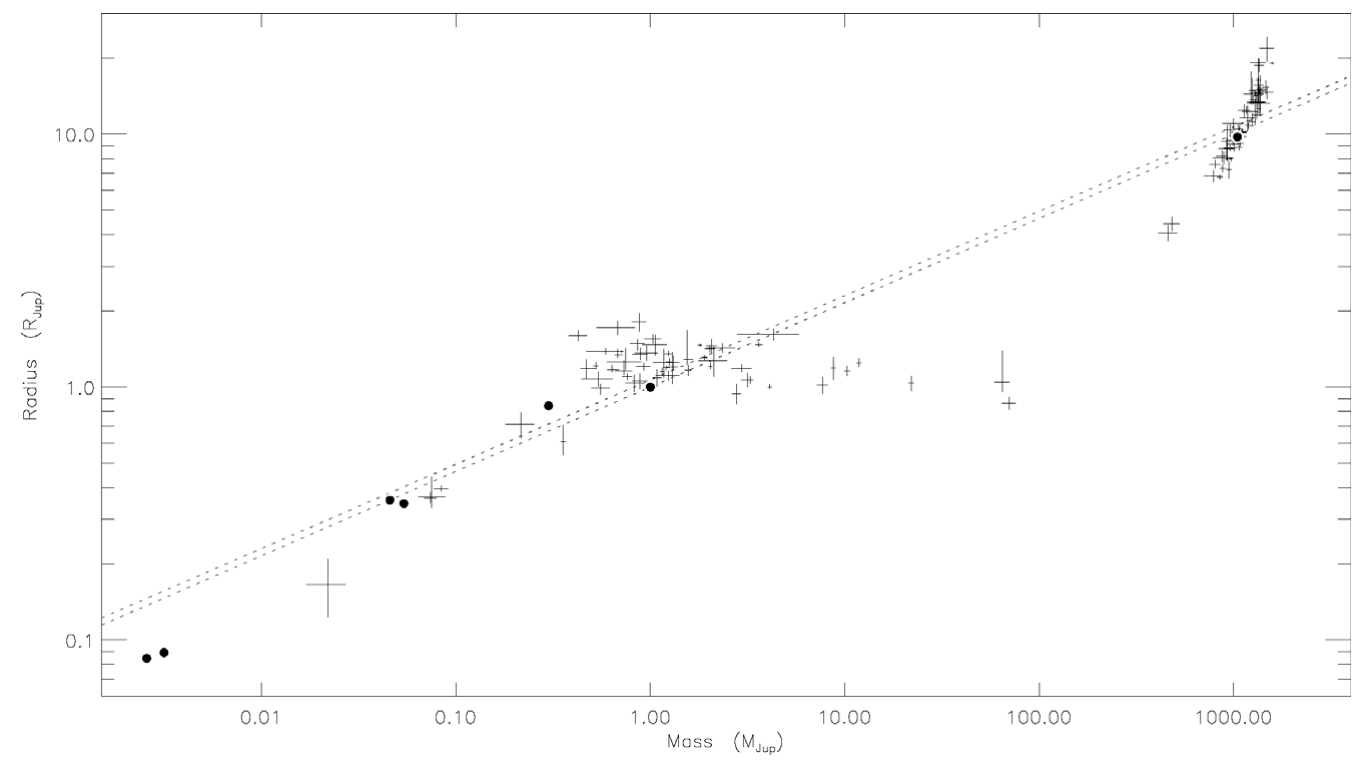

Figure 1. Mass-radius plot of the 58 systems studied within this project. Several Solar system bodies are shown by filled circles. Dashed lines show the densities of Jupiter and the Sun.

In Paper IV (Southworth 2011) I have extended the number of objects to 58, which includes 15 CoRoT systems, 10 observed by Kepler, five HAT discoveries, eight WASP objects, and all OGLE, TrES and XO planets. A plot of the masses and radii of the planets and their parent stars is shown in Fig. 1. I have also introduced an online catalogue of transiting planets, at http://www.astro.keele.ac.uk/ jkt/tepcat/. TEPCat contains the combined results from my project, a critical compilation of the physical properties of all known transiting planetary and brown-dwarf systems, and a summary of the basic observable quantities which are useful for planning follow-up observations.

\section{References}

Jenkins, J. M., Caldwell, D. A., \& Borucki, W. J. 2002, ApJ, 564, 495

Seager, S. \& Mallén-Ornelas, G. 2003, ApJ, 585, 1038

Southworth, J. 2008, MNRAS, 386, 1644 (Paper I)

Southworth, J. 2009, MNRAS, 394, 272 (Paper II)

Southworth, J. 2010, MNRAS, 408, 1689 (Paper III)

Southworth, J. 2011, MNRAS, in press, arXiv:1107.1235 (Paper IV)

Southworth, J. Bruntt, H., \& Buzasi, D. L. 2007, A\&A, 467, 1215

Southworth, J. Wheatley, P. J., \& Sams, G. 2007, MNRAS, 379, L11

Southworth, J. Maxted, P. F. L., \& Smalley, B. 2004a, MNRAS, 349, 547

Southworth, J. Maxted, P. F. L., \& Smalley, B. 2004b, MNRAS, 351, 1277

Southworth, J. Zucker, S., Maxted, P. F. L., \& Smalley, B. 2004c, MNRAS, 355, 986

Southworth, J. Maxted, P. F. L., \& Smalley, B. 2005a, A\&A, 429, 645

Southworth, J. Smalley, B., Maxted, P. F. L., Claret, A., \& Etzel, P. B. 2005b, MNRAS, 363, 529

Southworth, J. et al., 2009a, MNRAS, 396, 1023

Southworth, J. et al., 2009b, MNRAS, 399, 287

Southworth, J. et al., 2009c, ApJ, 707, 167

Southworth, J. et al., 2010, MNRAS, 408, 1680

Southworth, J. et al., 2011, A\&\&A, 527, A8

Torres, G., Winn, J. N., \& Holman, M. J., 2008, ApJ, 677, 1324 